\title{
PERFIL METABÓLICO DE PORTADORES DO HIV - HOSPITAL DE CLÍNICAS DE CURITIBA
}

\author{
METABOLIC PROFILE OF HIV-INFECTED PATIENTS - HOSPITAL DE CLÍNICAS \\ DE CURITIBA
}

Camila Cristina Valério ${ }^{1}$, Pamela Liberalino Bohnke ${ }^{1}$, Paola Wyatt Brock ${ }^{1}$, Eliane Carmes ${ }^{1}$, Miguel I. A. Hanna Sobrinho ${ }^{1}$

\section{RESUMO}

Objetivos: Avaliar e comparar o perfil lipídico e a glicemia de pacientes portadores do HIV em uso de inibidores de protease (IP) e de inibidores da transcriptase reversa não nucleosídeos (ITRNN). Material e Métodos: Série de casos. Elegíveis 126 pacientes HIV-positivo, transmissão horizontal, acompanhados, no mínimo, 24 meses no ambulatório de Infectologia HC-UFPR. Incluídos 52 pacientes que completaram 5 avaliações no serviço, quando foram coletadas informações sobre data do diagnóstico, esquema de tratamento, lipidograma, glicemia de jejum, contagem de células CD4, carga viral e comorbidades. Os pacientes que permaneceram em uso da mesma classe de antirretroviral foram separados em dois grupos: os que utilizavam ITRN-IP e os tratados com ITRN-ITRNN. Comparou-se as observações entre os grupos na primeira e na quinta consulta e, a evolução dos pacientes que completaram as cinco avaliações. Resultados: Na primeira avaliação, os grupos ITRN-IP $(n=18)$ e ITRN-ITRNN ( $n=34)$ foram semelhantes, exceto quanto aos valores médios de HDL-colesterol (mg/dL): 40,63 $\pm 10,18$ versus 50,28 $\pm 17,67, p=0,0249$, respectivamente. Na quinta avaliação, observou-se menores valores médios de triglicerídeos (mg/dL) no grupo ITRN-ITRNN, $145,16 \pm 83,13$, em relação ao ITRN-IP, 191,00 $\pm 82,63, p=0,0590$. E ainda, o nível sérico de LDL-colesterol foi menor do que $70 \mathrm{mg} / \mathrm{dL}$ somente entre $3(11,11 \%)$ pacientes do grupo ITRN-ITRNN, enquanto que no grupo ITRN-IP todos apresentaram LDL-colesterol maior do que $70 \mathrm{mg} / \mathrm{dL}, p=0,0459$. Conclusão: Neste estudo não foram observadas alterações significativas no perfil metabólico de pacientes sob TARV, em uso de IP ou ITRNN. Entretanto, observou-se perfil lipídico mais próximo da meta estabelecida para pacientes HIV-positivo naqueles em uso de regimes contendo ITRNN.

Palavras-chave: HIV; Dislipidemia

\section{ABSTRACT}

Aims: To analyse and compare the lipid profile and blood glucose of HIV-infected patients in use of protease inhibitors (PI) and reverse transcriptase inhibitors non-nucleoside (NNRTI). Methods: Case series. 126 eligible HIV-positive patients, horizontal transmission, followed at least 24 months in the infectious diseases service of HC-UFPR. Included 52 patients who completed 5 appointments in service. Informations were collected: date of diagnosis, treatment regimen, lipid profile, fasting glucose, CD4 cell count, viral load and comorbidities. Patients who remained on antiretroviral use of the same class were divided into two groups: those who used NRTI-IP and those treated with NRTIs-NNRTIs. Data were compared between the groups in the first and fifth consultation and analysed the outcome of patients who completed the five assessments. Results: In the first evaluation, the NRTI-IP group $(n=18)$ and NRTI-NNRTI group $(n=34)$ were similar, except for the mean values of HDL-cholesterol $(\mathrm{mg} / \mathrm{dL}): 40.63 \pm 10.18 \mathrm{vs}$. $50.28 \pm 17.67, p=0,0249$, respectively. In the fifth evaluation, there was lower mean triglyceride levels $(\mathrm{mg} / \mathrm{dL})$ in NRTI-NNRTI-group $145.16 \pm 83.13$, in relation to NRTI-IP, $191.00 \pm 82.63, p=0,0590$. Also, the level of serum LDL cholesterol was less than $70 \mathrm{mg} / \mathrm{dl}$ only between $3(11.11 \%)$ patients of the NRTI-NNRTI-group, while in the NRTI-IP group all showed higher LDL-cholesterol $70 \mathrm{mg} / \mathrm{dL}$, $p=0.0459$. Conclusion: In this study no significant changes were observed in the metabolic profile of patients on HAART in use of PI or NNRTI. However, there was nearest lipid profile of the target set for HIV-positive patients using NNRTI-containing regimens.

Key word: HIV; Dyslipidemia.

1-Universidade Federal do Paraná - UFPR

Contato/Mail to:

pamela_bohnke@hotmail.com

Rua General Carneiro, 181 - Alto da Glória, Curitiba - PR, 80060-900, 10 Andar Departamento Clínica Médica 


\section{INTRODUÇÃO}

Depois da introdução da terapia antirretroviral (TARV) para o tratamento de portadores do Vírus da Imunodeficiência Humana (HIV), observou-se acentuada queda das taxas de morbidade e mortalidade da Síndrome da Imunodeficiência Adquirida (SIDA) ${ }^{1}$. Cinco classes de medicamentos são aprovadas para o uso em infecções pelo HIV: inibidores da transcriptase reversa nucleosídeos (ITRN), inibidores da transcriptase reversa não nucleosídeos (ITRNN), inibidores da protease (IP), inibidores da integrase e inibidores de entrada do vírus. O esquema terapêutico mais utilizado é composto pelas drogas ITRN, ITRNN e $I P^{2}$.

Entre os efeitos secundários associados ao tratamento estão as alterações no perfil lipídico e na glicemia, que se tornaram evidentes com o aumento da sobrevida dos portadores do HIV, proporcionado pela TARV.

Além do uso de antirretrovirais, outros fatores têm sido associados às alterações metabólicas em portadores do HIV: fatores específicos do vírus, mecanismos inflamatórios relacionados com a infecção e/ou seu tratamento, ativação crônica do sistema imunológico e predisposição genética ${ }^{3}$. Ainda assim, a contribuição relativa à TARV e aos efeitos da própria doença na gênese dessas alterações não está bem definida na literatura. ${ }^{4}$

As alterações no perfil lipídico geralmente ocorrem entre o terceiro e o sexto mês do início da TARV, mas em regimes contendo IP podem ocorrer mais precoce e pronunciadamente ${ }^{5}$. A duração da exposição à TARV também tem sido associada ao desenvolvimento de alterações metabólicas.

Quanto às variações da glicemia, a hiperglicemia franca é mais raramente relatada entre os indivíduos tratados com antirretrovirais. Quando presente, está mais associada a regimes terapêuticos contendo IP $\mathrm{P}^{6}$.

O objetivo desse estudo foi avaliar e comparar o perfil lipídico e a glicemia de pacientes em uso de IP e de ITRNN.

\section{METODOLOGIA}

O estudo foi realizado no Serviço Ambulatorial de Infectologia do Hospital de Clínicas da Universidade Federal do Paraná (HC-UFPR), com revisão de 127 prontuários (126 incluídos na análise).

Elegíveis 126 pacientes HIV-positivo, transmissão horizontal, em acompanhamento por, no mínimo, 24 meses (entre 2007 e 2012). Incluídos no estudo 52 pacientes sob TARV que completaram 5 avaliações no serviço.

Foram registradas informações para definir o perfil do paciente: data de nascimento, sexo, data do diagnóstico da infecção pelo HIV e comorbidades: dislipidemia prévia, doença cardíaca, doença renal, distúrbio cognitivo, diabetes mellitus, hipertensão arterial, doenças pulmonares, tabagismo, etilismo, drogadição e neoplasias. A classificação do "Centers for Disease Control" (CDC) para adultos e adolescentes infectados pelo HIV também foi documentada. Essa classificação enfatiza a importância da contagem de linfócitos T CD4 + na categorização das condições clínicas relacionadas com o HIV e orienta as ações clínicas e terapêuticas no manejo de adolescentes e adultos infectados pelo HIV. O sistema baseia-se em três classes de contagem de linfócitos T CD4 + (1, 2 e 3) e três categorias clínicas ( $A, B$ e $C$ ) e está representada por uma matriz de nove categorias mutuamente exclusivas.

Para avaliar o efeito do tratamento, coletou-se informações de cinco consultas, com seis a oito meses de intervalo. Os dados obtidos foram: esquema da TARV, outros medicamentos utilizados, resultados de exames laboratoriais próximos às consultas (lipidograma, glicemia de jejum, contagem de células CD4, carga viral, sorologias), comorbidades. Os pacientes que permaneceram em uso da mesma classe de antirretroviral, durante as 5 avaliações, foram considerados grupo ITRN-IP $(n=18)$ quando tratados com IP e grupo ITRN-ITRNN ( $n=34$ ) quando com ITRNN. Os exames foram realizados no laboratório do $\mathrm{HC}$ UFPR. Para avaliar o perfil metabólico considerou-se dosagens de CT, LDL-C, HDL-c, TG e glicemia de jejum. Para calcular os valores do LDL-C, utilizou-se a fórmula de Friedewald, quando valores de TG menores que 400 $\mathrm{mg} / \mathrm{dl}$. Todos os valores foram registrados em $\mathrm{mg} / \mathrm{dl}$. Para determinar a atividade da doença considerou-se a contagem de células CD4 e a carga viral.

Para a análise estatística, utilizou-se os pacotes estatísticos Statistica 10 (StatSoft, Inc, Tulsa, OK, EUA) e MedCalc Statistical Software version 14.10.2 (MedCalc Software bvba, Ostend, Belgium).

Os dados foram sumarizados por média e desvio padrão, mediana e limites ou frequência e percentagem, conforme o tipo da variável. Para avaliar a significância das diferenças entre as observações conforme o tipo de tratamento, em cada uma das avaliações, foi utilizado, para variáveis quantitativas, o teste $t$ para amostras independentes, com transformação logarítmica para aquelas com distribuição assimétrica. Para as variáveis categóricas foi utilizado o teste do qui-quadrado ou o teste exato de Fisher, conforme recomendado.

Para as comparações entre a primeira e quinta avaliação, utilizou-se, para variáveis quantitativas, o 
teste $\mathrm{t}$ para amostras pareadas, com transformação logarítmica para aquelas com distribuição assimétrica. Para variáveis categóricas, o teste de McNemar. O nível de significância adotado foi $p<0,05$.

\section{RESULTADOS}

Foram analisados 52 pacientes, 28 homens $(53,85 \%)$, com média de idade na primeira avaliação de 39,4 anos. A média do tempo de doença foi de, aproximadamente, 3,6 anos. Quanto à gravidade da doença (classificação do $\mathrm{CDC}$ ), disponível para 31 pacientes; as classes mais frequentemente encontradas foram C3 (48,39\%) e A2 (19,35\%), conforme Tabela 1.

$\mathrm{Na}$ primeira avaliação (Tabela 2), os grupos ITRN-IP e ITRN-ITRNN foram semelhantes, exceto quanto aos valores médios de $\mathrm{HDL}$-colesterol $(\mathrm{mg} / \mathrm{dL})$ : $40,63 \pm 10,18$ versus $50,28 \pm 17,67, \quad p=0,0249$, respectivamente.

TABELA 1 - CARACTERÍSTICAS CLÍNICODEMOGRÁFICAS NA PRIMEIRA AVALIAÇÃO: PACIENTES INFECTADOS PELO HIV EM TRATAMENTO - SERVIÇO DE INFECTOLOGIA HC-UFPR - 2007 A 2012.

\begin{tabular}{lr}
\hline CARACTERÍSTICAS & \multicolumn{1}{c}{ VALORES } \\
\hline IDADE $^{(1)}(n=52)$ & $39,43 \pm 9,18$ \\
Média \pm DP & 19,$87 ; 64,54$ \\
Limites & $28(53,85)$ \\
SEXO MASCULINO [n(\%)] & \\
CLASSIFICAÇÃO CDC (n=31) & $3(9,68)$ \\
A1 [n(\%)] & $6(19,35)$ \\
A2 [n(\%)] & $2(6,45)$ \\
A3 [n(\%)] & $1(3,22)$ \\
B2 [n(\%)] & $3(9,68)$ \\
B3 [n(\%)] & $1(3,22)$ \\
C2 [n(\%)] & $15(48,39)$ \\
C3 [n(\%)] &
\end{tabular}

TEMPO DE DIAGNÓSTICO ${ }^{(1)}$ $(n=52)$

Média \pm DP

$3,57 \pm 3,84$

Limites
Na quinta avaliação (Tabela 3), observou-se menores valores médios de triglicerídeos $(\mathrm{mg} / \mathrm{dL})$ no grupo ITRN-ITRNN, 145,16 $\pm 83,13$, em relação ao ITRNIP $, 191,00 \pm 82,63, p=0,0590$. $E$ ainda, o nível sérico de LDL-colesterol foi menor do que $70 \mathrm{mg} / \mathrm{dL}$ somente entre $3(11,11 \%)$ pacientes do grupo ITRN-ITRNN, enquanto que no grupo ITRN-IP todos apresentaram LDL-colesterol maior do que $70 \mathrm{mg} / \mathrm{dL}, \mathrm{p}=0,0459$.

Quando se comparou a evolução entre a primeira e a quinta avaliação não se observou diferença significativa no perfil metabólico dos pacientes tanto do grupo ITRN-IP quanto do grupo ITRN-ITRNN (TABELAS 4 e 5).

A evolução do CT e do LDL-c, do Grupo ITRN-IP e Grupo ITRN-ITRNN, entre todas as consultas avaliadas, pode ser observada nas figuras 1 e 2, respectivamente.
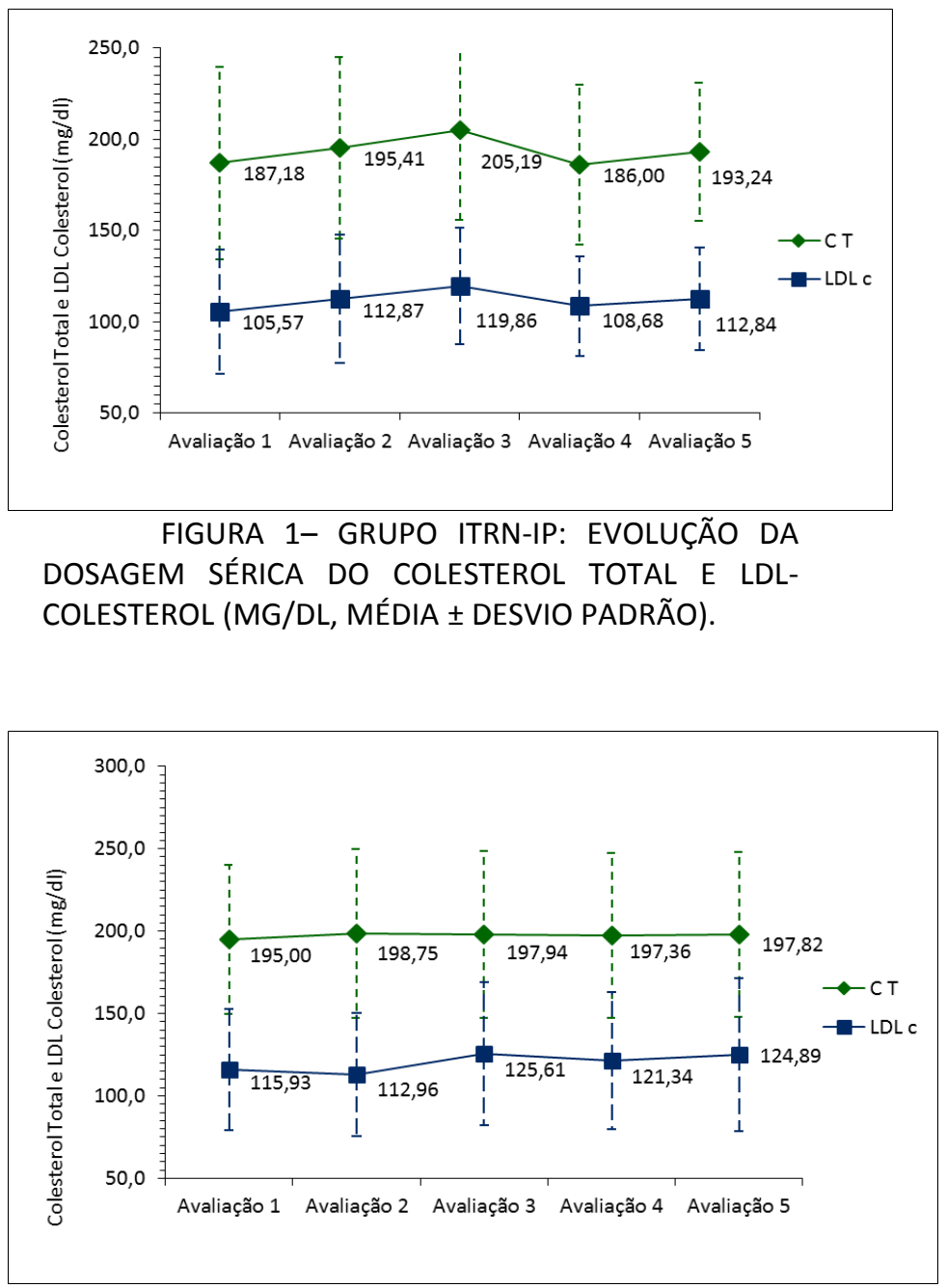

FIGURA 2 - GRUPO ITRN-ITRNN EVOLUÇÃO DA DOSAGEM SÉRICA DO COLESTEROL TOTAL E LDLCOLESTEROL (MG/DL, MÉDIA \pm DESVIO PADRÃO). 
TABELA 2 - COMPARAÇÃO ENTRE OS GRUPOS DE ACORDO COM USO DE ANTIRRETROVIRAL: CARACTERÍSTICAS CLÍNICO-DEMOGRÁFICAS NA PRIMEIRA AVALIAÇÃO - PACIENTES INFECTADOS PELO HIV EM TRATAMENTO - SERVIÇO DE INFECTOLOGIA HC-UFPR - 2007 A 2012.

\begin{tabular}{|c|c|c|c|}
\hline CARACTERÍSTICAS & $\begin{array}{c}\text { GRUPO ITRN- } \\
\text { IP } \\
\mathrm{N}(\%) \\
\end{array}$ & $\begin{array}{c}\text { GRUPO IITRN- } \\
\text { ITRNN } \\
\mathrm{N}(\%)\end{array}$ & $p$ VALOR \\
\hline IDADE & 18 & 34 & $0,6172^{(3)}$ \\
\hline Média $\pm \mathrm{DP}{ }^{(1)}$ & $38,55 \pm 10,49$ & $39,90 \pm 8,50$ & \\
\hline $\begin{array}{c}\text { TEMPO DE } \\
\text { DIAGNÓSTICO }\end{array}$ & 18 & 34 & $0,9809^{(3)}$ \\
\hline Média \pm DP ${ }^{(1)}$ & $3,55 \pm 3,81$ & $3,58 \pm 3,92$ & \\
\hline Mediana $^{(1)}$ & 1,39 & 1,85 & \\
\hline Limites $^{(1)}$ & 0,$12 ; 10,03$ & 0,$11 ; 14,07$ & \\
\hline CARGA VIRAL & 17 & 31 & $0,7470^{14}$ \\
\hline Detectável & $4(23,53)$ & $9(29,03)$ & \\
\hline Indectável & $13(76,47)$ & $22(70,97)$ & \\
\hline GLICEMIA & 15 & 30 & $0,9171^{(5)}$ \\
\hline Média $\pm D P^{(2)}$ & $\begin{array}{c}105,60 \pm \\
43,72\end{array}$ & $102,03 \pm 24,78$ & \\
\hline Mediana $^{(2)}$ & 94,00 & 99,50 & \\
\hline Limites $^{(2)}$ & 85,$00 ; 260,00$ & 72,$00 ; 219,00$ & \\
\hline COLESTEROL TOTAL & 17 & 33 & $0,5858^{(3)}$ \\
\hline Média $\pm \mathrm{DP}{ }^{(2)}$ & $\begin{array}{c}187,18 \pm \\
52,69\end{array}$ & $195,00 \pm 45,10$ & \\
\hline Mediana $^{(2)}$ & 175,00 & 191,00 & \\
\hline Limites $^{(2)}$ & $\begin{array}{l}118,00 ; \\
316,00\end{array}$ & 111,$00 ; 311,00$ & \\
\hline HDL-COLESTEROL & 16 & 29 & $0,0249^{(6)}$ \\
\hline Média $\pm D P^{(2)}$ & $40,63 \pm 10,18$ & $50,28 \pm 17,67$ & \\
\hline Mediana $^{(2)}$ & 41,00 & 45,00 & \\
\hline Limites $^{(2)}$ & 24,$00 ; 61,00$ & 23,$00 ; 95,00$ & \\
\hline LDL-COLESTEROL & 15 & 27 & $0,3768^{(3)}$ \\
\hline Média \pm DP ${ }^{(2)}$ & $\begin{array}{c}105,57 \pm \\
33,94\end{array}$ & $115,93 \pm 37,01$ & \\
\hline Mediana $^{(2)}$ & 99,40 & 109,20 & \\
\hline Limites $^{(2)}$ & 66,$80 ; 180,08$ & 48,$40 ; 212,60$ & \\
\hline $\begin{array}{c}\text { LDL-COLESTEROL < } \\
70[\mathrm{n}(\%)]\end{array}$ & $2(13,33)$ & $4(14,81)$ & $1,0000^{(7)}$ \\
\hline TRIGLICERÍDEOS & 16 & 32 & $0,2244^{(5)}$ \\
\hline Média $\pm \mathrm{DP}{ }^{(2)}$ & $\begin{array}{c}224,44 \pm \\
227,63\end{array}$ & $170,87 \pm 130,94$ & \\
\hline Mediana $^{(2)}$ & 172,00 & 144,00 & \\
\hline Limites $^{(2)}$ & $\begin{array}{c}76,00 ; \\
1044,00\end{array}$ & 53,$00 ; 673,00$ & \\
\hline
\end{tabular}

FONTE: os autores.

NOTA: (1) Valores em anos

(2) Valores em mg/dl

(3) Teste $t$ amostras independentes.

(4) Teste do qui-quadrado

(5) Teste $t$ amostras independentes, com transformação logarítmica.

(6) Teste $t$ amostras independentes, variâncias heterogêneas.

(7) Teste exato de Fisher.

DP: Desvio padrão; ITRN: inibidores da transcriptase reversa nucleosídeos; ITRNN: inibidores da transcriptase reversa não nucleosídeos; IP: inibidores da protease.
TABELA 3 - COMPARAÇÃO ENTRE OS GRUPOS DE ACORDO COM USO DE ANTIRRETROVIRAL: CARACTERÍSTICAS CLÍNICO-DEMOGRÁFICAS NA QUINTA AVALIAÇÃO - PACIENTES INFECTADOS PELO HIV EM TRATAMENTO - SERVIÇO DE INFECTOLOGIA HC-UFPR - 2007 A 2012.

\begin{tabular}{|c|c|c|c|}
\hline CARACTERÍSTICAS & $\begin{array}{c}\text { GRUPO ITRN-IP } \\
\mathrm{N}(\%)\end{array}$ & $\begin{array}{c}\text { GRUPO IITRN- } \\
\text { ITRNN } \\
\text { N (\%) }\end{array}$ & $p$ VALOR \\
\hline IDADE & 18 & 34 & $0,6250^{(3)}$ \\
\hline Média $\pm D^{(1)}$ & $41,82 \pm 10,30$ & $43,12 \pm 8,40$ & \\
\hline $\begin{array}{c}\text { TEMPO DE } \\
\text { DIAGNÓSTICO }\end{array}$ & 18 & 34 & $0,9847^{(3)}$ \\
\hline Média \pm DP ${ }^{(1)}$ & $6,82 \pm 4,19$ & $6,80 \pm 3,86$ & \\
\hline Mediana $^{(1)}$ & 4,62 & 5,79 & \\
\hline Limites $^{(1)}$ & 3,$02 ; 14,70$ & 2,$57 ; 18,31$ & \\
\hline CARGA VIRAL & 17 & 31 & $1,000^{(4)}$ \\
\hline Detectável & $1(5,88)$ & $2(6,45)$ & \\
\hline Indectável & $16(94,12)$ & $29(93,55)$ & \\
\hline GLICEMIA & 15 & 30 & $0,9596^{(5)}$ \\
\hline Média \pm DP ${ }^{(2)}$ & $99,00 \pm 24,45$ & $97,87 \pm 12,92$ & \\
\hline Mediana ${ }^{(2)}$ & 94,00 & 98,00 & \\
\hline Limites $^{(2)}$ & 79,$00 ; 158,00$ & 79,$00 ; 132,00$ & \\
\hline COLESTEROL TOTAL & 17 & 33 & $0,7418^{(3)}$ \\
\hline Média $\pm D^{(2)}$ & $193,24 \pm 37,87$ & $197,82 \pm 50,03$ & \\
\hline Mediana $^{(2)}$ & 187,00 & 194,00 & \\
\hline Limites $^{(2)}$ & 135,$00 ; 295,00$ & 120,$00 ; 318,00$ & \\
\hline HDL-COLESTEROL & 16 & 29 & $0,1546^{(3)}$ \\
\hline Média \pm DP ${ }^{(2)}$ & $41,01 \pm 11,89$ & $46,52 \pm 12,36$ & \\
\hline Mediana $^{(2)}$ & 38,00 & 43,00 & \\
\hline Limites $^{(2)}$ & 27,$00 ; 60,20$ & 28,$00 ; 66,00$ & \\
\hline LDL-COLESTEROL & 15 & 27 & $0,3039^{(6)}$ \\
\hline Média $\pm \mathrm{DP}^{(2)}$ & $112,84 \pm 28,01$ & $124,89 \pm 46,44$ & \\
\hline Mediana $^{(2)}$ & 106,80 & 120,20 & \\
\hline Limites $^{(2)}$ & 81,$60 ; 184,00$ & 44,$60 ; 214,80$ & \\
\hline $\begin{array}{c}\text { LDL-COLESTEROL }< \\
70[\mathrm{n}(\%)]\end{array}$ & $0(-)$ & $3(11,11)$ & $0,0459^{(4)}$ \\
\hline TRIGLICERÍDEOS & 16 & 32 & $0,0590^{(7)}$ \\
\hline Média \pm DP ${ }^{(2)}$ & $191,00 \pm 82,63$ & $145,16 \pm 83,13$ & \\
\hline Mediana $^{(2)}$ & 195,50 & 127,00 & \\
\hline Limites $^{(2)}$ & 62,$00 ; 317,00$ & 46,$00 ; 418,00$ & \\
\hline
\end{tabular}

FONTE: os autores.

NOTA: (1) Valores em anos

(2) Valores em $\mathrm{mg} / \mathrm{dl}$

(3) Teste $t$ amostras independentes.

(4) Teste exato de Fisher.

(5) Teste $t$ amostras independentes, com transformação logarítmica, variâncias heterogêneas.

(6) Teste $t$ amostras independentes, variâncias heterogêneas.

(7) Teste $t$ amostras independentes, com transformação logarítmica.

DP: Desvio padrão; ITRN: inibidores da transcriptase reversa nucleosídeos; ITRNN: inibidores da transcriptase reversa não nucleosídeos ; IP: inibidores da protease. 
TABELA 4 - GRUPO EM USO DE INIBIDORES DA PROTEASE: COMPARAÇÃO DAS CARACTERÍSTICAS CLÍNICAS ENTRE A PRIMEIRA E A QUINTA AVALIAÇÃO PACIENTES INFECTADOS PELO HIV EM TRATAMENTO SERVIÇO DE INFECTOLOGIA - HC-UFPR - 2007 A 2012.

\begin{tabular}{|c|c|c|c|}
\hline CARACTERÍSTICAS & AVALIAÇÃO 1 & AVALIAÇÃO 5 & $p$ VALOR \\
\hline $\begin{array}{l}\text { TEMPO ENTRE AS } \\
\text { AVALIAÇÕES (n) }\end{array}$ & \multicolumn{2}{|c|}{18} & \\
\hline Média $\pm D P^{(1)}$ & \multicolumn{2}{|c|}{$6,84 \pm 4,19$} & \\
\hline Mediana $^{(1)}$ & \multicolumn{2}{|c|}{4,62} & \\
\hline Limites $^{(1)}$ & \multicolumn{2}{|c|}{3,$02 ; 14,71$} & \\
\hline GLICEMIA (n) & \multicolumn{2}{|c|}{15} & $0,3718^{(3)}$ \\
\hline Média $\pm \mathrm{DP}(2)$ & $\begin{array}{c}105,60 \pm \\
43,72\end{array}$ & $99,00 \pm 24,44$ & \\
\hline $\begin{array}{l}\text { COLESTEROL TOTAL } \\
\text { (n) }\end{array}$ & \multicolumn{2}{|c|}{17} & $0,3704(3)$ \\
\hline Média $\pm D P^{(2)}$ & $\begin{array}{c}187,18 \pm \\
52,69\end{array}$ & $\begin{array}{c}193,23 \pm \\
37,87\end{array}$ & \\
\hline HDL-COLESTEROL (n) & \multicolumn{2}{|c|}{16} & $0,8557^{(4)}$ \\
\hline Média $\pm \mathrm{DP}(2)$ & $40,62 \pm 10,18$ & $41,01 \pm 11,89$ & \\
\hline LDL-COLESTEROL (n) & \multicolumn{2}{|c|}{15} & $0,3323^{(4)}$ \\
\hline Média $\pm \mathrm{DP}(2)$ & $\begin{array}{c}105,57 \pm \\
33,94\end{array}$ & $\begin{array}{c}112,84 \pm \\
28,01\end{array}$ & \\
\hline $\begin{array}{l}\text { LDL-COLESTEROL } \\
<70[\mathrm{n}(\%)]\end{array}$ & $2 / 15(13,33)$ & $0 / 15(-)$ & $0,2500^{(5)}$ \\
\hline TRIGLICERÍDEOS (n) & \multicolumn{2}{|c|}{16} & $0,8065^{(3)}$ \\
\hline Média $\pm \mathrm{DP}(2)$ & $\begin{array}{c}224,44 \pm \\
227,63\end{array}$ & $\begin{array}{c}191,00 \pm \\
82,63\end{array}$ & \\
\hline $\begin{array}{c}\text { CARGA VIRAL } \\
\text { DETECTÁVEL [n(\%)] }\end{array}$ & $4 / 17(23,53)$ & $1 / 17(5,88)$ & $0,5000^{(5)}$ \\
\hline
\end{tabular}

FONTE: os autores

NOTA: (1) Valores em anos

(2) Valores em $\mathrm{mg} / \mathrm{dl}$

(3) teste $t$ amostras pareadas, com transformação logarítmica

(4) teste $t$ amostras pareadas

(5) teste de McNemar

DP: desvio-padrão

\section{DISCUSSÃO}

Pacientes infectados pelo HIV estão sujeitos a um risco cardiovascular maior do que indivíduos não infectados. Estudos recentes demonstraram que as causas cardiovasculares foram responsáveis por $6,5 \%$ do total de mortes entre os pacientes infectados pelo HIV na América do Norte e Europa e por $8 \%$ na França ${ }^{7}$. Sabe-se que a própria doença contribui para o risco cardiovascular elevado, embora ainda não se conheça o mecanismo responsável por essa alteração. As principais hipóteses são invasão direta do miocárdio pelo HIV, disfunção endotelial, distúrbios metabólicos, fibrinólise comprometida, disfunção de citocinas, distúrbios autoimunes e alterações do sistema nervoso autônomo ${ }^{6,8}$. A TARV também está associada a fatores de risco cardiovascular como dislipidemia, resistência insulínica e diabetes mellitus ${ }^{9}$. Além disso, como a TARV fez da infecção pelo HIV uma condição crônica, é importante considerar as comorbidades relacionadas
TABELA 5 - GRUPO EM USO DE INIBIDORES DA TRANSCRIPTASE REVERSA NÃO NUCLEOSÍDEOS: COMPARAÇÃO DAS CARACTERÍSTICAS CLÍNICAS ENTRE A PRIMEIRA E A QUINTA AVALIAÇÃO - PACIENTES INFECTADOS PELO HIV EM TRATAMENTO - SERVIÇO DE INFECTOLOGIA - HC-UFPR - 2007 A 2012.

\begin{tabular}{|c|c|c|c|}
\hline CARACTERÍSTICAS & AVALIAÇÃO 1 & AVALIAÇÃO 5 & $p$ VALOR \\
\hline $\begin{array}{l}\text { TEMPO ENTRE AS } \\
\text { AVALIAÇÕES }(n)\end{array}$ & \multicolumn{2}{|c|}{34} & \\
\hline Média $\pm \mathrm{DP}^{(1)}$ & \multicolumn{2}{|c|}{$6,80 \pm 3,86$} & \\
\hline Mediana $^{(1)}$ & \multicolumn{2}{|c|}{5,79} & \\
\hline Limites $^{(1)}$ & \multicolumn{2}{|c|}{2,$57 ; 18,31$} & \\
\hline GLICEMIA (n) & \multicolumn{2}{|c|}{30} & $0,3549^{(3)}$ \\
\hline Média \pm DP ${ }^{(2)}$ & $\begin{array}{r}102,03 \pm \\
24,77\end{array}$ & $97,80 \pm 12,90$ & \\
\hline $\begin{array}{l}\text { COLESTEROL TOTAL } \\
\text { (n) }\end{array}$ & \multicolumn{2}{|c|}{33} & $0,5961^{(4)}$ \\
\hline Média $\pm \mathrm{DP}$ & $\begin{array}{r}195,00 \pm \\
45,10\end{array}$ & $\begin{array}{r}197,82 \pm \\
50,03\end{array}$ & \\
\hline HDL-COLESTEROL (n) & \multicolumn{2}{|c|}{29} & $0,2105^{(3)}$ \\
\hline Média $\pm \mathrm{DP}(2)$ & $50,26 \pm 17,66$ & $46,52 \pm 12,36$ & \\
\hline LDL-COLESTEROL (n) & \multicolumn{2}{|c|}{27} & $0,0901^{(4)}$ \\
\hline Média $\pm \mathrm{DP}{ }^{(2)}$ & $\begin{array}{r}115,93 \pm \\
37,01 \\
\end{array}$ & $\begin{array}{r}124,81 \pm \\
46,47\end{array}$ & \\
\hline $\begin{array}{l}\text { LDL-COLESTEROL } \\
<70[\mathrm{n}(\%)]\end{array}$ & $4 / 27(14,81)$ & $3 / 27(11,11)$ & $1,0000^{(5)}$ \\
\hline TRIGLICERÍDEOS (n) & \multicolumn{2}{|c|}{32} & $0,2733^{(3)}$ \\
\hline Média $\pm D P^{(2)}$ & $\begin{array}{r}170,87 \pm \\
130,94 \\
\end{array}$ & $\begin{array}{r}145,16 \pm \\
83,13 \\
\end{array}$ & \\
\hline $\begin{array}{l}\text { CARGA VIRAL } \\
\text { DETECTÁVEL }[n(\%)]\end{array}$ & $9 / 31(29,03)$ & $2 / 31(6,45)$ & $0,0233^{(5)}$ \\
\hline
\end{tabular}

FONTE: os autores

NOTA: (1) Valores em anos
(2) Valores em mg/dl
(3) Teste $t$ amostras pareadas, com transformação logarítmica
(4) Teste $t$ amostras pareadas
(5) Teste de McNemar
DP: Desvio padrão

ao avanço da idade que contribuem para o aumento do risco cardiovascular de portadores do vírus ${ }^{10}$.

Com relação às mudanças do perfil metabólico, a infecção pelo HIV causa um desequilíbrio no metabolismo dos lipídios, caracterizado por baixos níveis de HDL-C, LDL-c e CT, e altos níveis de $\mathrm{TG}^{1,5,6,11,12}$. Após a introdução do IP, os níveis de $\mathrm{HDL}-\mathrm{C}$ permanecem baixos e há aumento no CT, LDL-c e TG; enquanto que os regimes contendo ITRNN promovem aumento nas taxas de CT e LDL-C, e ainda, aumento concomitante dos níveis de HDL-c ${ }^{13}$. Neste estudo, na comparação entre os indivíduos em uso de ITRN-IP e ITRN-ITRNN, verificou-se que em média os valores de HDL-c foram menores, na primeira consulta e os de TG maiores na quinta consulta, nos pacientes em uso de ITRN-IP. Da mesma forma, Vilela e colaboradores encontraram valores alterados de HDL-c e de TG, e nenhuma diferença significativa em níveis de CT e LDL- 
c em pacientes em uso de IP quando comparados com indivíduos sem uso de $\mathrm{IP}^{14}$. Sabe-se que o HDL-c é um bom indicador do risco cardiovascular. Os regimes contendo ITRNN, portanto, estão relacionados a um perfil metabólico mais favorável do que os que contêm $\mathrm{IP}$, principalmente em pacientes com risco cardiovascular preexistente ${ }^{13}$.

As mudanças no perfil lipídico dependem da dose e do tempo de exposição à TARV ${ }^{1}$. Segundo Ceccato e colaboradores ${ }^{15}$, a duração do tratamento, tanto com IP como com ITRNN, aumenta significativamente a prevalência de dislipidemia e lipodistrofia, sendo mais acentuado nos pacientes em uso de IP. O estudo de Segerer e colaboradores ${ }^{16}$ demonstrou, no grupo em uso de IP, um aumento significativo das taxas de CT após três, seis e doze meses do início da TARV ( $p<0,001)$, em comparação com os valores registrados antes do início do tratamento. Os autores relataram ainda acréscimo de 25,5\% nos níveis de TG, no terceiro mês após início da TARV. Neste estudo, entretanto, os níveis de TG, CT e frações HDL-c e LDL-c se mantiveram estáveis no intervalo entre as cinco avaliações em ambos os grupos. Os resultados divergentes podem estar relacionados a variações na prevalência de dislipidemia - 28 a $80 \%$ - entres pacientes sob TARV. A hipertrigliceridemia (40-80\%) é mais prevalente do que a hipercolesterolemia (10-50\%) e formas mistas (10$40 \%)^{6}$. Esses efeitos também variam de acordo com as drogas antirretrovirais em uso e estão associados a outros fatores de risco ambientais e genéticos ${ }^{17}$.

Sabe-se que a estratificação de risco cardiovascular em pacientes infectados pelo HIV deve ser avaliada antes do início e durante a TARV. Cumpre destacar a importância do tratamento das alterações do perfil metabólico no controle do risco cardiovascular $^{7}$. A V Diretriz de Dislipidemias e Prevenção de Aterosclerose ${ }^{18}$ recomenda como meta de tratamento da dislipidemia, para pacientes de alto risco, o alcance de níveis de LDL-c inferiores a 70 $\mathrm{mg} / \mathrm{dL}$. Neste estudo, entre a primeira e a quinta avaliação, observou-se queda no número de pacientes de ambos os grupos que apresentavam LDL-c abaixo de $70 \mathrm{mg} / \mathrm{dL}$. No caso do grupo ITRN-IP, todos os indivíduos apresentaram LDL-c acima desse valor.

Em pacientes diabéticos, a doença cardiovascular é uma importante causa de morbidade e mortalidade ${ }^{19}$. Alterações no metabolismo da glicose são frequentes em pacientes infectados pelo HIV tratados com antirretrovirais; a hiperglicemia franca, porém, é mais raramente observada nesses indivíduos $^{6}$. A resistência insulínica foi demonstrada em $13 \%$ dos pacientes em um ano de uso de TARV ${ }^{20}$. Sabe-se que os IP aumentam a resistência insulínica, o risco de diabetes e, assim, o risco de doença cardiovascular $^{19}$. Neste estudo, foi analisada a variação da glicemia dos pacientes em uso de ITRN-IP e ITRNITRNN entre a primeira e a quinta avaliação, não sendo encontrada nenhuma alteração significativa entre os grupos. Apesar de existirem relatos na literatura de acréscimo nos níveis de glicemia de indivíduos sob TARV, os efeitos dessas drogas sobre o metabolismo de lipídeos são mais frequentes do que sobre a glicemia ${ }^{21}$. Algumas limitações devem ser consideradas na interpretação dos resultados deste estudo, pois é uma análise baseada em revisão de prontuários e apenas os dados que estavam disponíveis puderam ser analisados.

\section{CONCLUSÃO}

Neste estudo não foram observadas alterações significativas no perfil metabólico de pacientes sob TARV, em uso de IP ou ITRNN, durante o período considerado. Entretanto, da primeira à quinta avaliação, o número de pacientes que apresentavam $\mathrm{LDL}-\mathrm{c}<70 \mathrm{mg} / \mathrm{dL}$ sofreu queda importante, em ambos os grupos, porém, mais pronunciada no grupo em uso de IP. Observou-se, portanto, perfil lipídico mais próximo da meta estabelecida para pacientes HIVpositivo (V Diretriz de Dislipidemia e Prevenção de Aterosclerose) entre indivíduos em uso de regimes contendo ITRNN se comparados às terapias com IP.

A dislipidemia, a elevação da glicose sérica e outras alterações metabólicas devem ser pesquisadas e manejadas com atenção nos pacientes infectados pelo HIV. A estratificação de risco cardiovascular antes do início e durante a TARV é mandatória no seguimento desses indivíduos, não só pelos riscos inerentes à infecção pelo HIV e à TARV, mas também pela maior longevidade que a TARV proporciona a esses pacientes.

\section{REFERÊNCIAS}

1.Calza L, Manfredi R, Chiodo F. Hyperlipidaemia in patients with HIV-1 infection receiving highly active antiretroviral therapy: epidemiology, pathogenesis, clinical course and management. Int. J. Antimicrob. Agents. 2003; 22(2):89-99.

2.Garg H, Joshi A, Mukherjee D. Cardiovascular Complications of HIV Infection and Treatment. Cardiovascular \& Hematological Agents in Medicinal Chemistry. 2013;58-66.

3.Vu CN, Ruiz-esponda R, Yang E, Chang E, Gillard B, Pownall $\mathrm{HJ}$, et al. Altered relationship of plasma triglycerides to $\mathrm{HDL}$ cholesterol in patients with HIV/HAART-associated dyslipidemia: Further evidence for a unique form of Metabolic Syndrome in HIV 
patients. Metabolism [Internet]. Elsevier Inc.; 2013;62(7):1014-20.

4. Rose H, Hoy J, Woolley I, Tchoua U, Bukrinsky M, Dart A, et al. HIV infection and high density lipoprotein metabolism. Atherosclerosis [Internet]. 2008 Jul [cited 2013 Nov 11];199(1):79-86.

5.Sprinz E, Lazzaretti RK, Kuhmmer R, Ribeiro JP. Dyslipidemia in HIV-infected individuals. Braz. J. Infect. Dis. [Internet]. [cited 2013 Nov 19];14(6):575-88.

6. Calza L, Manfredi R, Pocaterra D, Chiodo F. Risk of premature atherosclerosis and ischemic heart disease associated with HIV infection and antiretroviral therapy. J. Infect. [Internet]. 2008 Jul [cited 2013 Nov 25];57(1):16-32.

7. Boccara F, Lang S, Meuleman C, Ederhy S, MaryKrause $M$, Costagliola $D$, et al. HIV and coronary heart disease: time for a better understanding. J. Am. Coll. Cardiol. [Internet]. Elsevier Inc.; 2013 Feb 5 [cited 2013 Nov 11];61(5):511-23.

8. Sani MU. Myocardial disease in human immunodeficiency virus (HIV) infection: a review. Wien. Klin. Wochenschr. [Internet]. 2008 Jan [cited 2013 Nov 25];120(3-4):77-87.

9. Kramer AS, Lazzarotto AR, Sprinz E, Manfroi WC. Alterações metabólicas, terapia antirretroviral e doença cardiovascular em idosos portadores de HIV. Arq. Bras. Cardiol. [Internet]. Arquivos Brasileiros de Cardiologia; 2009 Nov [cited 2013 Nov 25];93(5):561-8. 10. Torres TS, Cardoso SW, Velasque LDS, Marins LMS, Oliveira MS De, Veloso VG, et al. Aging with HIV: an overview of an urban cohort in Rio de Janeiro (Brazil) across decades of life. Braz. J. Infect. Dis. [Internet]. 2013 [cited 2013 Nov 19];17(3):324-31.

11. Egaña-Gorroño L, Martínez E, Cormand B, Escribà T, Gatell J, Arnedo M. Impact of genetic factors on dyslipidemia in HIV-infected patients starting antiretroviral therapy. AIDS [Internet]. 2013 Feb 20 [cited 2013 Nov 19];27(4):529-38.

12. Parrinello CM, Landay AL, Hodis HN, Gange SJ, Norris PJ, Young $M$, et al. Association of subclinical atherosclerosis with lipid levels amongst antiretroviraltreated and untreated HIV-infected women in the Women' $s$ Interagency HIV study. Atherosclerosis [Internet]. Elsevier Ltd; 2012;225(2):408-11.

13. Fontas $E$, van Leth $F$, Sabin CA, Friis-Møller $N$, Rickenbach $M$, d'Arminio Monforte $A$, et al. Lipid profiles in HIV-infected patients receiving combination antiretroviral therapy: are different antiretroviral drugs associated with different lipid profiles? J. Infect. Dis. [Internet]. 2004 Mar 15 [cited 2013 Nov 25];189(6):1056-74.

14. Vilela FD, Lorenzo AR de, Tura BR, Ferraiuoli GI, Hadlich M, Barros MV de L, et al. Risk of coronary artery disease in individuals infected with human immunodeficiency virus. Braz. J. Infect. Dis. [Internet]. [cited 2013 Nov 25];15(6):521-7.
15. Ceccato M, Bonolo P. Antiretroviral therapyassociated dyslipidemia in patients from a reference center in Brazil. Brazilian J. Med. Biol. Res. [Internet]. 2011 [cited 2013 Nov 26].

16. Segerer S, Bogner JR, Walli R, Loch O, Goebel FD. Hyperlipidemia under treatment with proteinase inhibitors. Infection [Internet]. [cited 2013 Nov 26];27(2):77-81.

17. Caron-Debarle M, Boccara F, Lagathu C, et al. Adipose tissue as a target of HIV-1 antiretroviral drugs. Potential consequences on metabolic regulations. Curr Pharm Des. 2010;16:3352-60.

18. Xavier HT, Izar MC, Faria Neto JR, Assad MH, Rocha VZ, Sposito AC, et al. V Diretriz Brasileira de Dislipidemias e Prevenção da Aterosclerose. Arq. Bras. Cardiol. [Internet]. Arquivos Brasileiros de Cardiologia; 2013 [cited 2013 Nov 11];101(4):01-22.

19. Thienemann F, Sliwa K, Rockstroh JK. HIV and the heart: the impact of antiretroviral therapy: a global perspective. Eur. Heart J. [Internet]. 2013 Oct 14 [cited 2013 Nov 22].

20. Pinto Neto LF da $S$, das Neves MB, RibeiroRodrigues R, Page K, Miranda AE. Dyslipidemia and fasting glucose impairment among HIV patients three years after the first antiretroviral regimen in a Brazilian AIDS outpatient clinic. Braz. J. Infect. Dis. [Internet]. [cited 2013 Nov 28];17(4):438-43.

21. Tsiodras S, Mantzoros C, Hammer S, Samore M. Effects of protease inhibitors on hyperglycemia, hyperlipidemia, and lipodystrophy: a 5-year cohort study. Arch. Intern. Med. [Internet]. 2000 Jul 10 [cited 2013 Nov 28];160(13):2050-6. 